\title{
Establishing Prospective IVIVC for Generic Pharmaceuticals: Methodologies Assessment
}

\author{
Sumon Chakraborty*, Kavan Pandya and Deepika Aggarwal
}

Centre of Excellence-Biostudies, Integrated Product Development Organization, Dr. Reddy's Laboratories, Hyderabad, Andhra Pradesh, India

\begin{abstract}
The present study was aimed to establish prospective IVIVC method for generic products using example of two different drug formulations (aprepitant capsules, immediate release and donepezil tablets, sustained release). The in vitro dissolution of these formulations was examined by using USP-II apparatus and different range of dissolution media. The dissolution profile was matched with the deconvoluted profile of drugs obtained from literature data to select biorelevant dissolution media. An IVIVC was established by using the mean fraction dissolved (FD) and mean fraction absorbed (FA) and used to simulate the plasma profile of these formulations by convolution from optimized dissolution media. The in vivo drug disposition was studied in an open label, balanced, randomized, single dose, two way crossover study in healthy subjects. Predicted PK parameters were compared with observed parameters. A positive correlation was seen between the FD and FA for both formulations with $\mathrm{r}^{2}=0.989$ for aprepitant and $\mathrm{r}^{2}=0.995$ for donepezil. The percent prediction error for both $\mathrm{C}_{\max }$ and $\mathrm{AUC}_{\mathrm{t}}$ were $\leq 15 \%$ while predicting the plasma concentration time profile for human bioequivalence studies for these formulations. Results supports use of prospective method in establishing IVIVC while predicting in vivo pharmacokinetic profile for bioequivalence studies for generic product development.
\end{abstract}

Keywords: Convolution, deconvolution, aprepitant, donepezil, IVIVC.

\section{INTRODUCTION}

In vitro-in vivo correlation (IVIVC) is a predictive mathematical model describing the relationship between an in vitro property and a relevant in vivo response of a drug. Dissolution testing of dosage forms has extensively been used for quality control, ensuring batch to batch similarity, formulation discrimination etc. $[1,2]$. Such dissolution testing does not assure bioequivalence (BE). However in vitro dissolution in biorelevant media has been used for setting up IVIVC and in vitro bioequivalence for certain class of drugs $[3,4]$. The IVIVC has its own significance in product designing, changing or scaling up, setting up dissolution specification with respect to in vivo behavior of formulation [5-7].

For a generic product to come into existence, bioequivalence needs to be proven against the innovator. The prime strategy of generic formulation development is to deliver a product having similar in vivo performance (plasma profile) as that of innovator product. In order to achieve the sameness, formulator needs to know the influence of various formulation changes on in vivo outcome of the formulation. In vivo performance can be predicted from dissolution testing (in vitro) using In vitro in vivo correlation (IVIVC). IVIVC has mainly two elements: convolution and deconvolution.

*Address correspondence to this author at the Centre of ExcellenceBiostudies, Integrated Product Development Organization, Dr. Reddy's Laboratories, Bachupally, Hyderabad, Andhra Pradesh, India; Tel: +91 404334647; Fax: +91 404346285; E-mail: sumonc@drreddys.com
The convolution approach assures direct correlation of dissolution data and pharmacokinetic parameters $\left(\mathrm{C}_{\max }, \mathrm{T}_{\max }\right)$. Predicting plasma concentration time course in a single step using convolution method is relatively simple when compared to the two stage methodology in deconvolution model (Fig. 1) [8]. Classical Level A IVIVC is not possible always due to the extensive data requirement (three formulations and IV/IR data) [6], which would not be completely available during developmental phase of drug product. A recent article by Gillespie also reiterated that convolution IVIVC models afford certain advantages over the deconvolution models [9].

The use of different type of IVIVC definitely affects strategic decision in the generic pharmaceutical development. For a generic company, establishing complete IVIVC using deconvolution approach would be expensive, complex and time consuming. On the other hand, prospective IVIVC would be much easier and efficient in predicting bioequivalence metrics from reference and to test in vitro dissolutions.

Thus, the objective of this article is to exemplify the significance of prospective IVIVC to its application during generic product development especially during strategy development of the product. We have performed BE for two different generic pharmaceuticals (aprepitant and donepezil) and have correlated the observed results with the predicted results from convolution method.

\section{MATERIAL AND METHODS}

\section{Dosage Forms}

Two different formulations, one containing $125 \mathrm{mg}$ of aprepitant and other having $23 \mathrm{mg}$ of donepezil were used in 


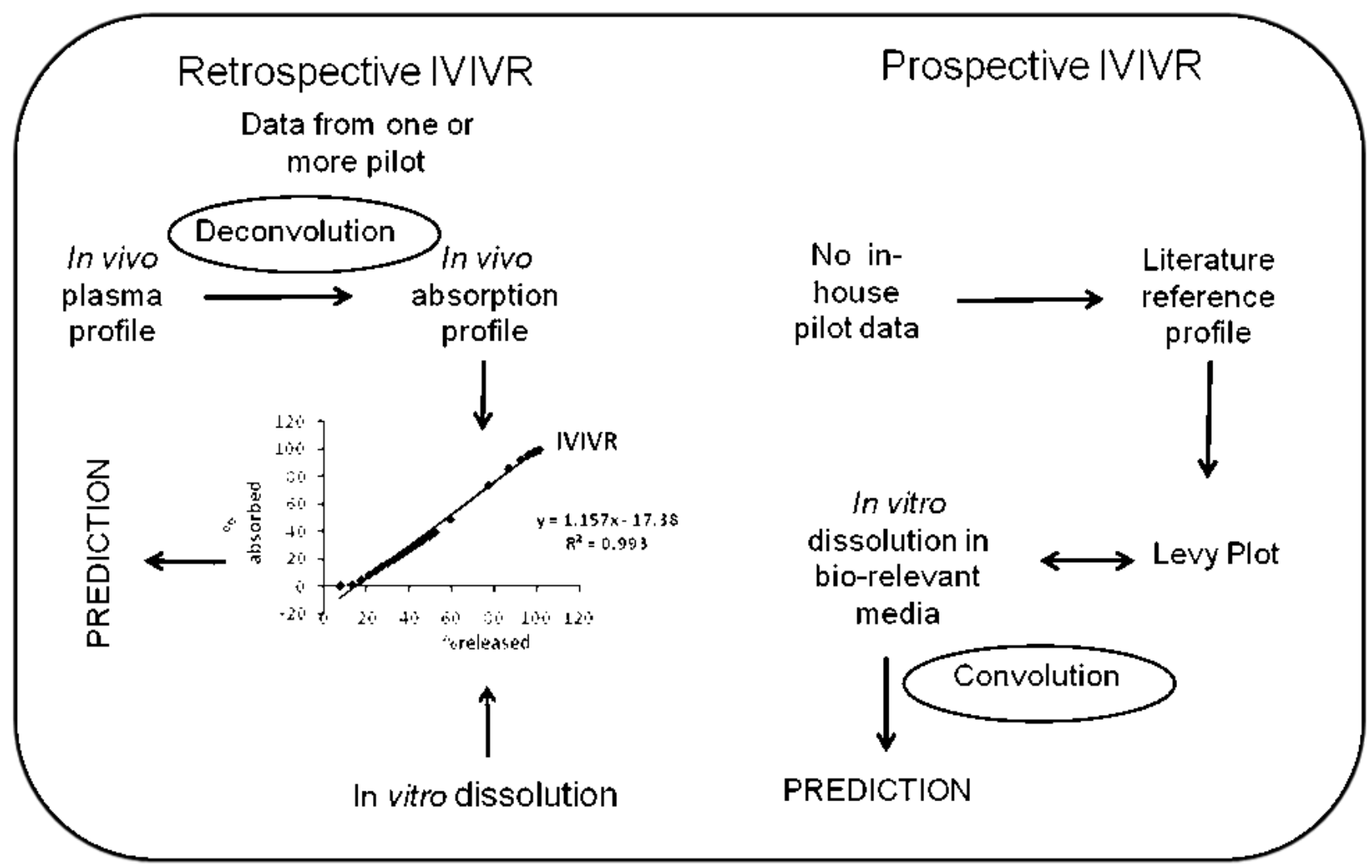

Fig. (1). Schematic representation of retrospective and prospective IVIVC approaches for predicting pharmacokinetic parameters prior to human bioequivalence studies for generic products.

this study. The first formulation was an immediate release (IR) aprepitant capsule containing (w/w) 30\% aprepitant, $6 \%$ hydroxypropyl cellulose, $0.1 \%$ SLS, $30 \%$ sucrose, $15 \%$ MCC (18.9\% hard gelatin capsule shell weight). The technology used for the formulation of aprepitant capsules was drug nanoparticles coated over MCC beads (Wet milling of drug dispersion to achieve particle size diameter of less than $400 \mathrm{~nm}$ ). Hydroxypropyl cellulose, aprepitant and SLS were added in the purified water and stirred for 20 minutes, and the suspension was milled using bead mill until getting desired particle size. Then the drug suspension was added in the sucrose solution. Drug loading was carried out using the above prepared suspension by bottom spray over celphere (MCC). Drug loaded pellets were lubricated with SLS for 5 minutes and filled in a capsule filling machine [Dr. Reddy's Laboratory, Batch \# F015]. The reference for aprepitant capsules used in the study was commercially available as EMEND capsules [Merck and Co., INC, USA, Batch \# 193027]. The other formulation was a sustained release donepezil tablet containing (w/w) 12\% donepezil, 38\% lactose, 21\% Eudragit L 100 55, 23\% HPMC K100 LV CR, $2.7 \%$ each of low grade HPC and HPC (EXF) and $0.6 \%$ magnesium stearate [Dr. Reddy's Laboratory, Batch \# EC 10261]. The reference for donepezil tablets was commercially available as Aricept tablets [Eisai Inc., Batch \# 004837].

\section{In Vivo Testing}

Only healthy subjects having normal health conditions determined by medical history and physical examination were selected for the study. The age of the subjects included in bioequivalence study (aprepitant/donepezil) was between $18-45$ years and weighing at least $50 \mathrm{Kg}(52-70 \mathrm{Kg})$ for both BE. Selected routine clinical laboratory measurements were performed during screening. Informed consent was obtained from subjects after explaining the purpose and the nature of the study. Subjects were instructed to abstain from taking any medication for one week prior to and during the study period. Also, it was ensured that the subjects had not consumed any alcohol and had not smoked for $48 \mathrm{~h}$ before drug administration and throughout period of the sample collection.

Aprepitant: An open label, balanced, randomized, single dose, two way crossover study was conducted in 18 healthy subjects to compare the bioequivalence of test product [Dr. Reddy's Laboratory $125 \mathrm{mg}$ aprepitant capsules] and reference product [Emend, Merck and CO, INC, USA]. All the study protocols were reviewed and approved by Independent review boards, IRB. The study was conducted under fasting conditions with a washout period of 10 days. The drug was administered in fasting subjects $(\sim 10 \mathrm{~h}$ overnight fasting) with $240 \mathrm{~mL}$ of water. Blood samples were obtained from each subject before dosing and at 1.0, $2.0,3.0,4.0,5.0,6.0,7.0,8.0,9.0,10.0,11.0,12.0,13.0$, 14.0, 16.0, 18.0, 20.0, 24.0, 48.0 and $72.0 \mathrm{~h}$ post-dose. Plasma was isolated from the whole blood and stored at -20 ${ }^{\circ} \mathrm{C}$ until analysis. The concentration of Aprepitant was quantitated at Dr. Reddy's Laboratories Ltd., India using LC-MS / MS method, which is validated according to the international guidelines. 
Donepezil: This was a randomized, single dose two way crossover, bioequivalence study of two formulation of donepezil tablet [Test: Dr. Reddy's Laboratory $23 \mathrm{mg}$ tablet and Reference: Aricept manufactured by Eisai Inc.]. This study was conducted in normal, healthy, adult human subjects under fasting conditions with a washout period of 28 days. All the study protocols were reviewed and approved by Independent review boards [IRB]. Both test and reference drugs were administered in fasting subjects $(\sim 10 \mathrm{~h}$ overnight fasting) with $240 \mathrm{~mL}$ of water. Blood samples were collected at 1.0, 2.0, 3.0, 4.0, 5.0, 6.0, 7.0, 8.0, 9.0, 10.0, 11.0, 12.0, 13.0, 14.0, 16.0, 18.0, 20.0, 24.0, 48.0 and $72.0 \mathrm{~h}$ post-dose. Plasma was isolated by centrifugation and analyzed for concentration of donepezil at Dr. Reddy's Laboratories Ltd., India using LC-MS / MS method, which is validated according to the international guidelines.

\section{Pharmacokinetic Parameters}

Mean plasma concentration time profiles for each of the formulation were constructed using WINNONLIN version 5.2 software. Maximum plasma concentration $\left(\mathrm{C}_{\max }\right)$ was determined by direct inspection of the mean plasma concentration time profile. The time at which $\mathrm{C}_{\max }$ occurred was the $\mathrm{T}_{\max }$. AUC from time 0 to $\mathrm{t}(\mathrm{AUCt})$ was determined by trapezoidal method. The area under the plasma concentration versus time curve from time zero to infinity was calculated using following formula, $\mathrm{AUCinf}=\mathrm{AUC} 0-\mathrm{t}$ $+\mathrm{Ct} / \lambda \mathrm{z}, \mathrm{Ct}$ is the last measurable concentration and $\lambda \mathrm{z}$ is the terminal elimination rate constant. The elimination rate constant was determined for each of the molecule by linear regression of the linear portion of the curve [ $1 \mathrm{n}$ (concentration versus time curve)].

\section{Dissolution Testing}

Aprepitant: In vitro dissolution studies were performed using USP-II (paddle apparatus) at $50 \mathrm{rpm}$ in the following bio relevant dissolution media: (a) Water with $0.5 \%$ SLS, (b) $6.5 \mathrm{pH}$ FaSSIF and (c) $6.5 \mathrm{pH}$ FaSSIF where sodium taurocholate $(\mathrm{NaTc})$ and lecithin replaced by $0.25 \%$ SLS. Twelve capsules were used in the dissolution experiment. Each capsule was dipped in the above mentioned media (500 $\mathrm{ml}$ for first two media and $900 \mathrm{ml}$ for the final media) at $37^{\circ} \mathrm{C}$. Concentrations of aprepitant were measured by UV spectrophotometry at $0,5,10,15,20,30,45,60,90$ and 120 minutes in fasted state simulated intestinal fluid (FaSSIF) and $0,5,10,15,20,30,45,60$ minutes in Water with $0.5 \%$ SLS. Cumulative amount release of aprepitant at these time points was calculated.

Donepezil: USP-II (paddle) apparatus at $50 \mathrm{rpm}$ was used for in vitro dissolution testing with $900 \mathrm{ml}$ of following bio relevant dissolution media: (a) 2.1 SGF without SLS, (b) $0.1 \mathrm{~N} \mathrm{HCl}$, (c) $4.5 \mathrm{pH}$ sodium phosphate buffer, (d) $5.5 \mathrm{pH}$ sodium phosphate buffer and at $25 \mathrm{rpm}$ in $900 \mathrm{ml}$ of $0.1 \mathrm{HCl}$ $(2 \mathrm{~h})$ followed by $6.8 \mathrm{pH}$ phosphate buffer. For dissolution experiment, 12 tablets were used and each tablet was dipped into the above mentioned media at $37{ }^{\circ} \mathrm{C}$. The concentration of donepezil was measured by UV spectrophotometry at 0,1 , $2,3,4,5,6,7,8,9,10,12$ and $14 \mathrm{~h}$. Cumulative amount release of donepezil at this time points was calculated.

\section{IVIVC}

The IVIVC was developed using the mean in vitro dissolution data (percentage dissolve) and mean in vivo dissolution data (percentage absorb in vivo). Fraction of drug absorbed to the systemic circulation was obtained by deconvoluting the plasma profile by numerical deconvolution method $[6,10]$. In this method, time course of the drug input is estimated by using a mathematical model based on convolution integral.

$C_{t}=\int_{0}^{t} C \delta(t-u)$ rabs $u(d u)$

From above equation, $r_{a b s}$ is absorption rate time course, $\mathrm{C}_{\delta}$ represents the concentration time profile resulting from an instantaneous absorption of a unit amount of drug which is typically from bolus or infusion intravenous injection or reference oral solution data (in this case intravenous infusion), $C_{t}$ represents the plasma concentration versus time profiles of the tested formulations and $u$ is the variable of integration.

At each time point, mean FD (Fraction dissolved) was plotted versus mean FA (fraction absorbed) in a levy plot. The slope, intercept and correlation coefficient describing the relationship between mean FD and mean FA were determined using linear regression.

Aprepitant: In general, FaSSIF media is used to assess the in vitro dissolution of poorly soluble drug which is correlated with in vivo performance. However amount of sodium taurocholate and lecithin may be insufficient for certain poorly soluble drugs such as aprepitant and hence there is need to develop a simplified version of FaSSIF with common surfactants used in dissolution keeping other ingredients same. Aprepitant follows one compartment pharmacokinetic model, so from the literature plasma profile [11] pharmacokinetic profile of $125 \mathrm{mg}$ of aprepitant was deconvoluted to get in vivo dissolution profile. $2 \mathrm{mg}$ intravenous infusion (for 4 hours) of aprepitant was used to calculate the unit impulse response (UIR) [11]. In vitro dissolution profiles were generated in (FaSSIF) and FaSSIF where sodium taurocholate (NaTC) and lecithin were replaced by $0.1 \%, 0.25 \%$ and $0.5 \%$ SLS. All the dissolution media were similar to FaSSIF in terms of osmolarity and buffer capacity. Dissolution rate and extent were very slow in FaSSIF so NaTC and lecithin were replaced by SLS. Levy plots were used to optimize the media and from these media results, PK parameters were computed by convolution technique and were validated in human bioequivalence study of test formulation versus reference formulation (emend) in healthy human subjects. Ratios of test and reference formulation for $\mathrm{C}_{\max }$ and $\mathrm{AUC}$ were computed and compared with predicted ratios.

Donepezil: Donepezil follows two compartment pharmacokinetic models, so from in-house data pharmacokinetic profile of reference formulation [12] was deconvoluted to get in vivo dissolution profile. In house IR data of $10 \mathrm{mg}$ were used as UIR for the SR product. Deconvoluted profile was compared with the in vitro dissolution profile in different dissolution media and the particular dissolution profile was selected which is super imposable to the doconvoluted profile for further 
convolution in order to get the predicted plasma profile for $23 \mathrm{mg}$ SR donepezil.

Prediction error: The prediction error ( $\%$ PE) calculation was used to access quantitatively, how accurate a given model can predict a pharmacokinetic parameter for a drug. The \% PE of both $\mathrm{C} \max$ and AUC was calculated using the same formula. In this formula, the observed value is subtracted from the predicted values in the model. This resulting value is then divided by the observed value. The value is then converted to a percentage to by multiplying by 100.

$$
\begin{aligned}
& \% P E C_{\max }=\left[\left(C_{\text {max obs }}-\left(C_{\text {max pred }}\right)\right] / C_{\text {maxobs }} * 100\right. \\
& \% \text { PE AUC }=\left[(A U C)_{\text {obs }}-(A U C)_{\text {pred }}\right] / A U C_{\text {obs }} * 100
\end{aligned}
$$

\section{RESULTS AND DISCUSSION}

\section{Aprepitant}

The mean values of $\mathrm{C}_{\max }, \mathrm{AUC}_{\mathrm{t}}$ and $\mathrm{T}_{\max }$ after oral administration of the immediate release EMEND capsules extracted form literature sources were $1539 \mathrm{ng} / \mathrm{mL}$ and $19455 \mathrm{ng} / \mathrm{mL}$ for $1 \mathrm{~h}$ and $4 \mathrm{~h}$ respectively. These values were taken from the SBOA of aprepitant approved by US FDA [13].

The mean in vitro dissolution profile for aprepitant IR capsule (EMEND, $125 \mathrm{mg}$ ) along with the deconvoluted profile is shown in Fig. (2). Dissolution profile in $0.25 \%$ SLS in FaSSIF is similar and super imposable on the deconvoluted profile of aprepitant.

The IVIVC was established using in vitro dissolution data and in vivo dissolution data (Deconvoluted profile). Linear regression analysis in the levy plot showed (Fig. 3) that a statistically significant relationship $\left(r^{2}=0.989\right)$ existed between the FD and FA for aprepitant reference formulation in $0.25 \%$ SLS with FaSSIF media.

So this media was further explored as bio relevant media. Based on the dissolution profile obtained in $0.25 \%$ SLS with FaSSIF media, pharmacokinetic parameters were predicted for test and reference formulation by convolution method and test to reference ratio was calculated for $C_{\max }$ and $\mathrm{AUC}_{\mathrm{t}}$

The comparison of predicted and observed PK parameters, $\mathrm{T} / \mathrm{R}$ ratio and the $\% \mathrm{PE}$ for test and reference formulations have been tabulated in Table 1. From these results, it is evident that the prospective convolution using the optimized biorelevant media was able to successfully predict the in vivo PK parameters with a minimal $(<15 \%)$ prediction error. Hence, for poorly soluble drug like aprepitant, there is a need of critical evaluation in selecting bio relevant media with appropriate modification which will further help to achieve IVIVC.

\section{Donepezil}

The mean values of $\mathrm{C}_{\max }, \mathrm{AUC}_{\mathrm{t}}$ and $\mathrm{T}_{\max }$ for sustained release Aricept tablets were $32.63 \mathrm{ng} / \mathrm{mL}$ and $1561.46 \mathrm{ng} / \mathrm{mL}$ for $1 \mathrm{~h}$ and $6.15 \mathrm{~h}$, respectively [12].

The mean in vitro dissolution profile for donepezil SR tablet (Aricept) along with the deconvoluted profile is shown in Fig. (4). From the in vitro dissolution profile of Aricept, $0.1 \mathrm{~N} \mathrm{HCl}$ followed by $6.8 \mathrm{~PB}$ was able to closely describe the in vivo absorption profile and thus it was used for IVIVC for donepezil.

The IVIVC was established using in vitro dissolution data and in vivo dissolution data (Deconvoluted profile).

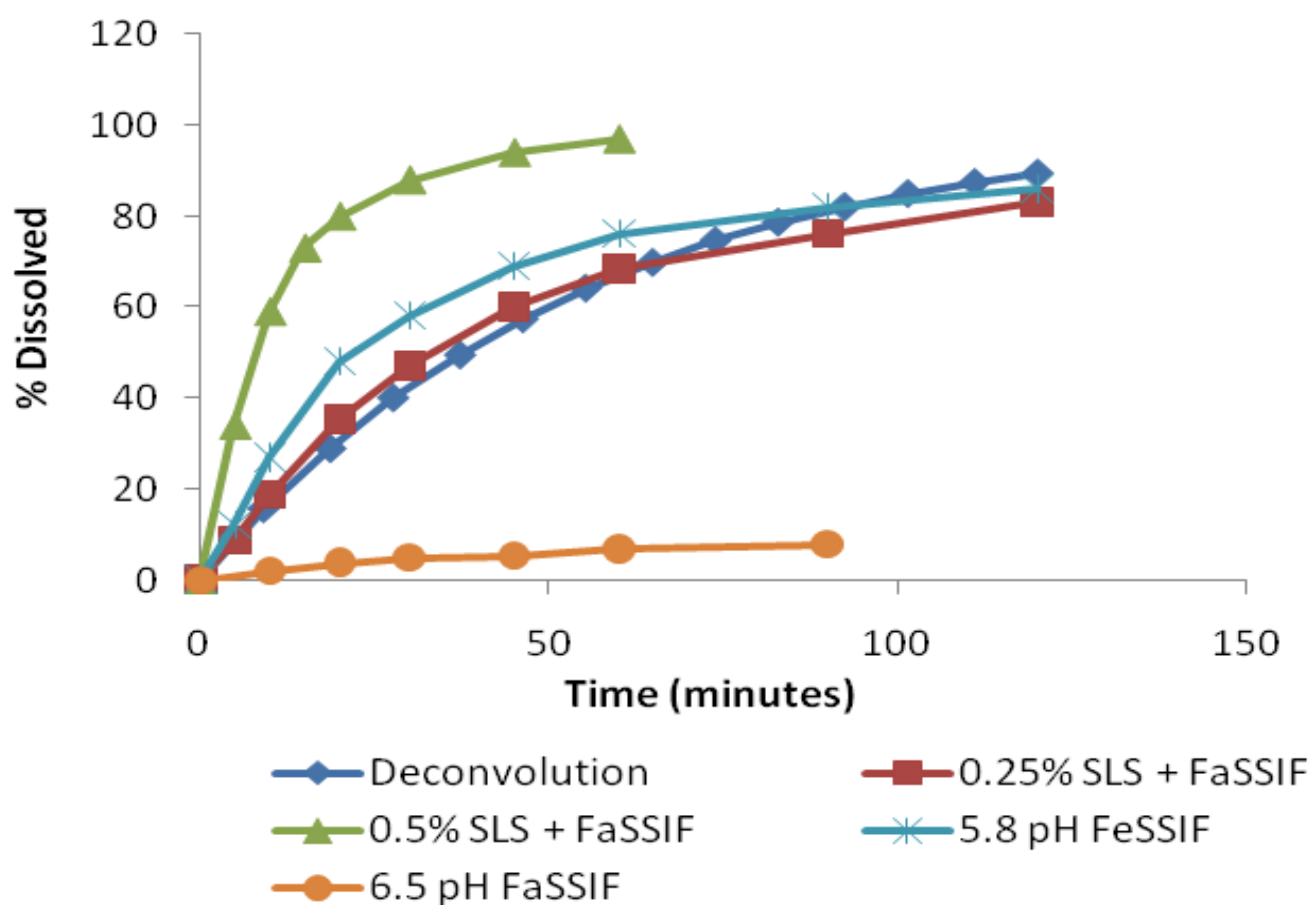

Fig. (2). In vitro dissolution profile along with the deconvoluted profile of EMEND in USP-II (paddle apparatus) at $50 \mathrm{rpm}$ speed. Each time point represents the mean fraction dissolved of twelve capsules of EMEND (aprepitant, $125 \mathrm{mg}$ ). 


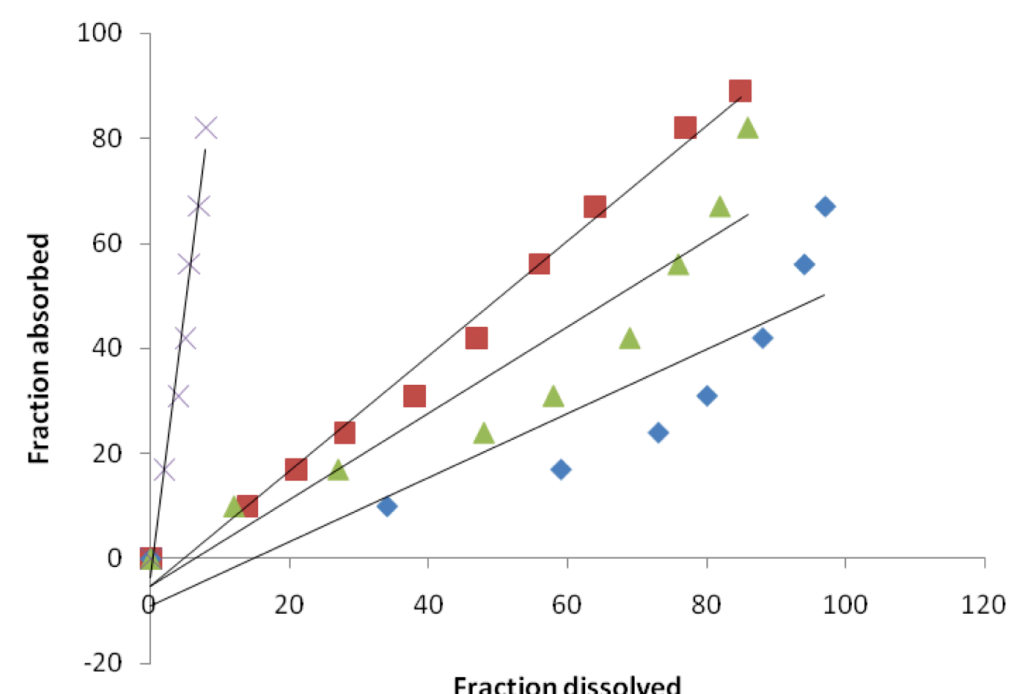

\begin{tabular}{llcc}
\hline & Dissolution media & Linear trend line equation & $\mathrm{r}^{2}$ value \\
\hline$\square$ & $0.25 \%$ SLS+ FaSSIF & $\mathrm{y}=1.097 \mathrm{x}-5.395$ & $\mathrm{R}^{2}=0.989$ \\
\hline \multirow{4}{*}{$0.5 \%$ SLS+ FaSSIF } & $\mathrm{y}=0.610 \mathrm{x}-9.193$ & $\mathrm{R}^{2}=0.798$ \\
$\times$ & $5.8 \mathrm{pH} \mathrm{FeSSIF}$ & $\mathrm{y}=0.822 \mathrm{x}-5.276$ & $\mathrm{R}^{2}=0.886$ \\
\hline & $6.5 \mathrm{pH} \mathrm{FaSSIF}$ & $\mathrm{y}=10.18 \mathrm{x}-3.679$ & $\mathrm{R}^{2}=0.977$ \\
\hline
\end{tabular}

Fig. (3). Relationship between the mean fraction absorbed in vivo and the mean fraction dissolved in vitro for EMEND. Mean FD and mean FA were determined for the reference formulation and used to establish the IVIVC. The line represents the linear regression of the data.

Table 1. Prediction of Pharmacokinetic Parameters (Cmax and AUCt) for Reference and Test Formulation of Aprepitant IR Capsule (125 mg) by Numerical Convolution Method Using Winnonlin Version 5.2 IVIVC Toolkit as an Simulation Software and the Corresponding Prediction Error

\begin{tabular}{|c|c|c|c|c|c|c|}
\hline Formulation & $\begin{array}{c}\mathrm{C}_{\max } \text { Observed } \\
(\mathrm{ng} / \mathrm{mL})\end{array}$ & $\underset{(\mathrm{ng} / \mathrm{mL})}{\mathrm{C}_{\max } \text { Predicted }}$ & $\% \mathrm{PE}$ & $\begin{array}{c}\text { AUC }_{\mathrm{t}} \text { Observed } \\
(\mathrm{ng} / \mathrm{mL})\end{array}$ & $\begin{array}{c}\text { AUC }_{\mathrm{t}} \text { Predicted } \\
(\mathrm{ng} / \mathrm{mL})\end{array}$ & $\% \mathrm{PE}$ \\
\hline Reference & 1735 & 1539 & $11 \%$ & 41828 & 46757 & $11 \%$ \\
\hline Test & 1378 & 1275 & $7 \%$ & 35368 & 39336 & $11 \%$ \\
\hline $\mathrm{T} / \mathrm{R}$ & $79.42 \%$ & $84.40 \%$ & & $84.50 \%$ & $85.00 \%$ & \\
\hline
\end{tabular}

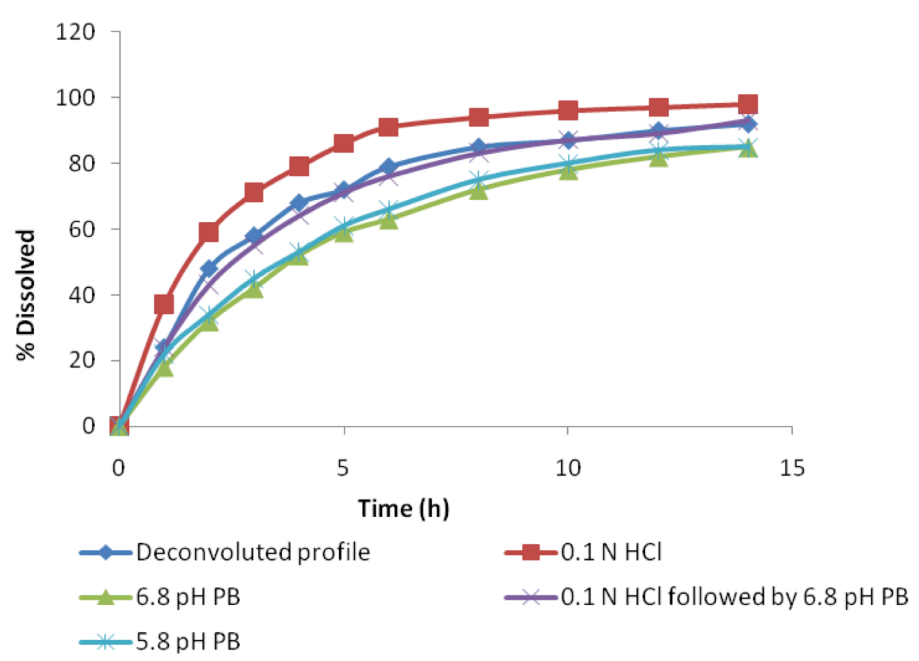

Fig. (4). In vitro dissolution profile along with the deconvoluted profile of Aricept in USP-II (paddle apparatus) at 50 rpm speed. Each time point represents the mean of fraction dissolved of twelve tablets. 


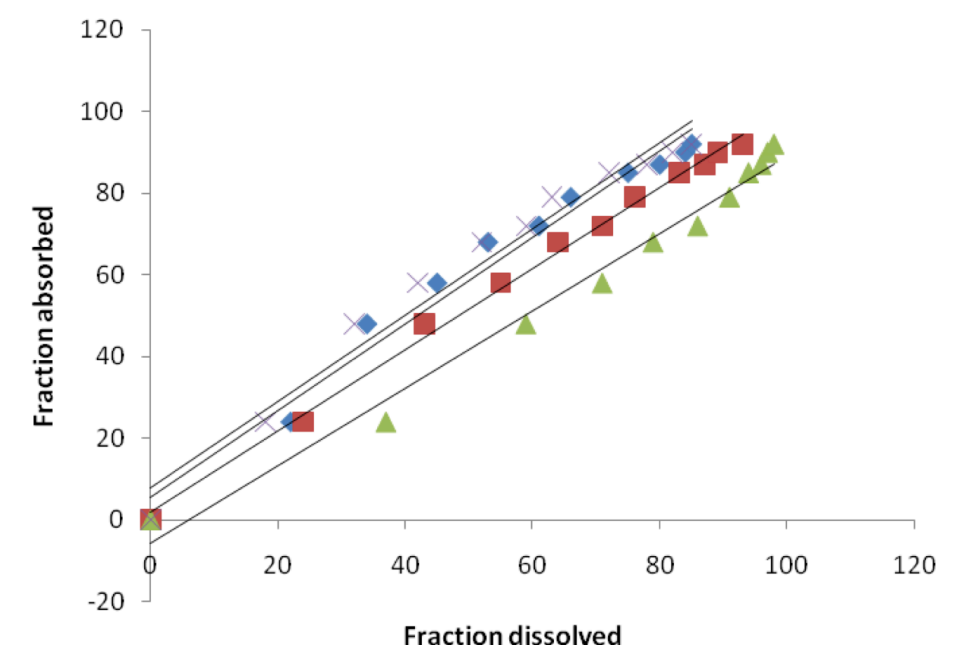

\begin{tabular}{llrr}
\hline \multicolumn{1}{c}{ Dissolution media } & Linear trend line equation & $\mathrm{r}^{2}$ value \\
\hline $\mathbf{\Delta}$ & $0.1 \mathrm{~N} \mathrm{HCl}$ & $\mathrm{y}=0.949 \mathrm{x}-5.825$ & 0.983 \\
$\times$ & $6.8 \mathrm{pH}$ Phosphate buffer & $\mathrm{y}=1.056 \mathrm{x}+7.918$ & 0.970 \\
$\mathbf{0}$ & $\mathrm{y}=0.997 \mathrm{x}+1.806$ & 0.995 \\
$\quad 5.5 \mathrm{HCl}$ followed by $6.8 \mathrm{pH}$ phosphate buffer & $\mathrm{y}=1.059 \mathrm{x}+5.659$ & 0.975 \\
\hline
\end{tabular}

Fig. (5). Relationship between the mean fraction absorbed in vivo and the mean fraction dissolved in vitro for Aricept. Mean FD and mean FA were determined for the reference formulation and used to establish the IVIVC. The line represents the linear regression of the data.

Table 2. Prediction of Pharmacokinetic Parameters (Cmax and AUCt) for Reference and Test Formulation of Donepezil SR Tablet (23 mg) by Numerical Convolution Method Using Winnonlin Version 5.2 IVIVC Toolkit as an Simulation Software and the Corresponding Prediction Error

\begin{tabular}{|c|c|c|c|c|c|c|}
\hline Formulation & $\begin{array}{c}\mathrm{C}_{\max } \text { Observed } \\
(\mathrm{ng} / \mathrm{mL})\end{array}$ & $\begin{array}{c}\mathrm{C}_{\max } \text { Predicted } \\
(\mathrm{ng} / \mathrm{mL})\end{array}$ & $\% \mathrm{PE}$ & $\begin{array}{c}\text { AUC }_{\mathrm{t}} \text { Observed } \\
(\mathrm{ng} / \mathrm{mL})\end{array}$ & $\begin{array}{l}\text { AUC }_{\mathrm{t}} \text { Predicted } \\
(\mathrm{ng} / \mathrm{mL})\end{array}$ & $\% \mathrm{PE}$ \\
\hline Reference & 28.19 & 29 & 2.87 & 1543.18 & 1601.82 & 3.75 \\
\hline Test & 25.8 & 27.9 & 8.1 & 1501.81 & 1541.78 & 2.7 \\
\hline $\mathrm{T} / \mathrm{R}$ & $91.52 \%$ & $96.21 \%$ & & 97.32 & 96.25 & \\
\hline
\end{tabular}

Linear regression analysis in the levy plot showed (Fig. 5) a statistically significant relationship $\left(\mathrm{r}^{2}=0.995\right)$ between the $\mathrm{FD}$ and $\mathrm{FA}$ for donepezil reference formulation in $0.1 \mathrm{~N} \mathrm{HCl}$ followed by 6.8 phosphate buffer media.

So this media was further explored as bio relevant media. Based on the dissolution profile obtained in $0.1 \mathrm{~N} \mathrm{HCl}$ followed by 6.8 phosphate buffer, pharmacokinetics parameters $\left(\mathrm{C}_{\max }\right.$ and $A U C_{t}$ ) were predicted for both formulations by convolution method and test to reference ratio was calculated for $\mathrm{C}_{\max }$ and AUC $_{\text {t }}$. Table 2 shows the comparison of predicted and observed ratios along with the $\% \mathrm{PE}$.

From the above results, it is clear that using prospective convolution in an optimized biorelevant media we can closely predict the in vivo PK parameters with a low prediction error.

\section{CONCLUSION}

This study clearly advocates the benefit of prospective IVIVC method using an optimized biorelevant media for predicting PK parameters for human bioequivalence for a generic product. The in vivo Cmax and AUCt were predicted by calculating $\mathrm{Fa}$ from the IVIVC. In both examples (aprepitant and donepezil) in vivo plasma profiles were closely predicted $(\mathrm{PE} \%<15 \%)$. This further fortifies the fact that the in vitro dissolution of drugs in biorelevant media was similar to in vivo absorption profile of both drugs. This study provides an easy prospective convolution approach for predicting and establishing IVIVC for generic product development.

\section{CONFLICT OF INTEREST}

All the authors are employees of Dr. Reddy's laboratory and have no competing interest to declare.

\section{ACKNOWLEDGEMENTS}

Authors would like to thank Dr. Reddy's Laboratory for providing assistance and support to publish this work.

\section{REFERENCES}

[1] Brown, C.K.; Friedel, H.D.; Barker, A.R. FIP/AAPS joint workshop report: dissolution/in vitro release testing of novel/special dosage forms. Indian J. Pharm. Sci., 2011, 73(3), 338-353. 
[2] Khalid, S.; Gauhar, S.; Rehman, R.; Fatima, S. Post market surveillance of different brands of ofloxacin $200 \mathrm{mg}$ tablets available in local market of karachi (Pakistan). Int. J. Pharm. Sci., 2012, 4, 668-672

[3] Jantratid, E.; Maio V.D.; Ronda, E.; Dressman, J.B. Application of biorelevant dissolution tests to the prediction of in vivo performance of diclofenac sodium from an oral modified-release pellet dosage form. Eur. J. Pharm. Sci., 2009, 37, 434-441.

[4] Sunesen, V.H.; Pedersen, B.L.; Kristensen, H.G.; Müllertz, A. In vivo in vitro correlations for a poorly soluble drug, danazol, using the flow-through dissolution method with biorelevant dissolution media. Eur. J. Pharm. Sci., 2005, 24, 305-313.

[5] Sakore, S.; Chakraborty, B. In vitro-in vivo correlation (IVIVC): a strategic tool in drug development. J. Bioequiv. Avail., 2011, 1-12.

[6] Emami, J. In vitro - in vivo correlation: from theory to applications. J. Pharm. Sci., 2006, 9(2), 169-189.

[7] FDA, Guidance for industry. Extended release oral dosage forms: Development, evaluation and application of In vitro/In vivo correlations. U.S. Department of Health and Human Service, Food and Drug Administration, Center for Drug Evaluation and Research (CDER): USA, 1997.
[8] Bonlokke, L.; Hovgaard, L.; Kristensen, H.G.; Knutson, L.; Lindahl, A.; Lennernas, H. A comparison between direct determination of in vivo dissolution and the deconvolution technique in humans. Eur. J. Pharm. Sci., 1999, 8, 19-27.

[9] Gillespie, W.R.; Veng-Pedersen, P. Gastro-intestinal bioavailability: determination of in vivo release profiles of solid dosage forms by deconvolution. Biopharm. Drug Dispos., 1985, 6(3), 351-355.

[10] Grbic, S.; Parojcic, J.; Ibric, S.; Djuric, Z. In vitro-in vivo correlation for gliclazide immediate-release tablets based on mechanistic absorption simulation. AAPS. PharmSciTech., 2011, 12(1), 165-171.

[11] Majumdar, A.K.; Howard, L. Pharmacokinetics of aprepitant after single and multiple oral doses in healthy volunteers. J. Clin. Pharmacol., 2006, 46, 291-300.

[12] Clinical pharmacology and biopharmaceutics review of donepezil SR tablet $(23 \mathrm{mg})$. Center for Drug Evaluation and Research, Application number: 022568, Eisai Medical Research Inc., 2009.

[13] Clinical pharmacology and biopharmaceutics review of aprepitant capsule $(125 \mathrm{mg})$. Center for Drug Evaluation and Research, Application number: 21-549, Merck Research Laboratory, 2003.

(C) Chakraborty et al.; Licensee Bentham Open.

This is an open access article licensed under the terms of the Creative Commons Attribution Non-Commercial License (http://creativecommons.org/licenses/by-nc/ 3.0/) which permits unrestricted, non-commercial use, distribution and reproduction in any medium, provided the work is properly cited. 